\title{
Doubly Magic Nickel
}

\section{Two independent experiments on the isotope copper-79 confirm that its nuclear neighbor nickel-78 is indeed a doubly magic nucleus.}

\section{by Daniel Bazin*}

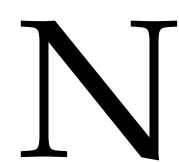

uclear physicists could easily pass for magicians. They often talk about magic nuclei as if they were about to pull these objects out of a hat and show their fuzzy long ears. This funny qualifier was coined by Eugene Wigner, who believed nuclei behave like uniform liquid droplets, but had to admit the experimental evidence pointed out by Maria Goeppert-Mayer that nuclei with neutron $(N)$ or proton $(Z)$ numbers $2,8,20,28$, 50,82 , and 126 were more stable than their neighbors [1]. Goeppert-Mayer and other physicists went on to explain this phenomenon on the basis of the nuclear shell model, in which protons and neutrons fill a nucleus in energy shells, or orbitals, akin to the layers of an onion. Magic numbers correspond to the greatest gaps in energy between shells, giving extra stability to nuclei in which those shells are filled completely [2]. When both the number of protons and of neutrons fulfills this requirement, the nucleus is called doubly magic.

Two new studies-one by Louis Olivier at the National Institute of Nuclear and Particle Physics (IN2P3-CNRS), France, and colleagues [3], and another by Andree Welker from CERN, Switzerland, and Technische Universität Dresden, Germany, and colleagues [4]-have now confirmed that nickel-78 ( $Z=28, N=50)$, a candidate doubly magic nucleus, is indeed a member of this exclusive club of nuclei. The finding is significant because, with almost two thirds of its matter composed of neutrons, nickel-78 is radioactive and one of the most neutron-rich doubly magic nuclei to date. Magic nuclei are benchmarks of the shell model, but because nickel-78 is so neutron-rich, physicists expect its structure to differ from that of its stable cousins and to be used to test the limits of this model.

The nuclear shell model has been very successful in describing most of the stable nuclei, which follow the sequence of magic numbers nicely. However, with the experimental advances brought, in large part, by the era of radioactive beams, experimenters have identified radioactive nuclei that have gone rogue and refuse to follow the normal sequence

\footnotetext{
* National Superconducting Cyclotron Laboratory, Michigan State University, East Lansing, MI 48824-1321, USA
}

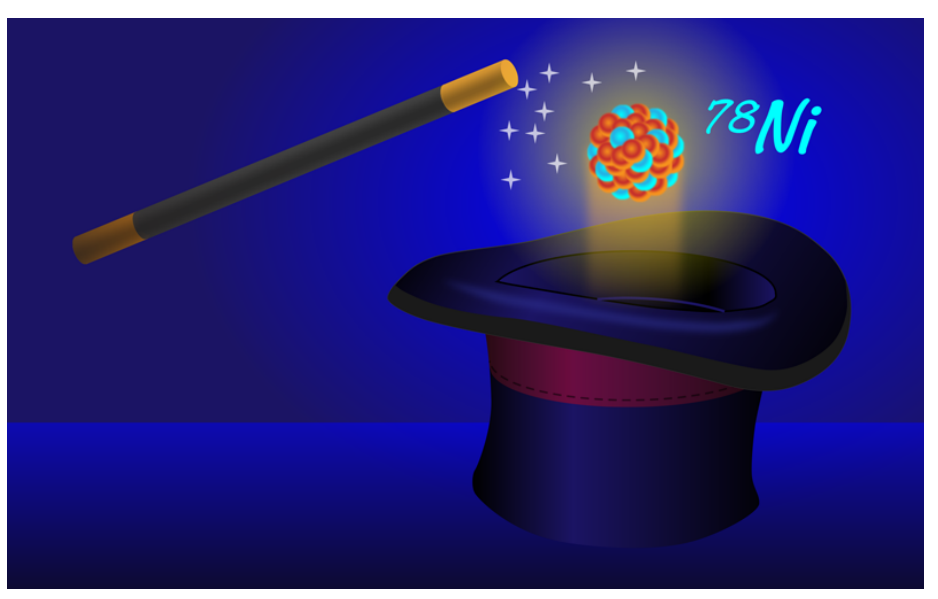

Figure 1: With 28 protons and 50 neutrons, nickel-78 is one of the most neutron-rich doubly magic nuclei known to date. Because it is difficult to produce in the laboratory and short-lived, physicists have to rely on clever tricks to study its properties. Olivier et al. [3] and Welker et al. [4] have used two different tricks to confirm that nickel-78 is a doubly magic nucleus. (APS/Alan Stonebraker)

[5]. The reason behind this behavior is rooted in the evolution of the orbitals' energies as the balance between the number of protons and neutrons changes. In the shell model, the nucleus is actually described by two separate sets of shells, one for protons and one for neutrons. Protons and neutrons are not identical particles and can therefore have the same quantum numbers. But protons and neutrons are both fermions, due to their spin of $1 / 2$, so they cannot share the same set of quantum numbers within their own shells. As more neutrons are added to the isotopic chain of an element, for instance, only the neutron shell is filled, and the proton-neutron component of the strong force steers the energies of the orbitals, either closing gaps by bringing the orbitals together or opening new gaps by repelling them from each other. The net result is the appearance and disappearance of magic numbers depending on the size of these gaps. One famous example of this phenomenon happens in the chain of oxygen isotopes, in which the first doubly magic nucleus is oxygen- $16(Z=8, N=8)$. However, the next one is not oxygen-28 $(N=20)$, as one might expect, but is instead oxygen-24 $(N=16)[6,7]$. In fact, oxygen-24 is the last known bound isotope of oxygen for the same reason 
that oxygen-24 displaces oxygen-28: the normal $N=20$ gap has shrunk and a new $N=16$ gap has opened, resulting in a binding energy of oxygen-28 that is too small to keep its nucleons bound together.

The chain of nickel isotopes $(Z=28)$ seems more in line with tradition. Nickel-56 $(N=28)$ is doubly magic and the next expected one is nickel-78 $(N=50)$. Nickel-78 is very difficult to produce in the laboratory, but the new studies of Olivier and co-workers and of Welker and co-workers have now managed to get close enough to this isotope. The two groups studied copper-79, which has only one proton more than nickel-78, and obtained experimental data suggesting that, unlike in the oxygen isotopes, the orbital energies in nickel-78 are not modified enough to close the $Z=28$ and $N=50$ gaps and remove its doubly magic character. The methods used by the two teams are very different, which strengthens the case even more.

Olivier and colleagues used a nuclear reaction known as a knockout reaction, which removed a proton from a high-speed zinc- 80 projectile that was produced at the Radioactive Ion Beam Factory (RIBF) facility in Tokyo, Japan. Such reactions bear this name because they happen so suddenly that the projectile can be considered frozen in time during the reaction. The resulting copper-79 nucleus can be excited to a state with higher energy than its ground state, and the probability of finding the nucleus in a given final state depends directly on which shell the ejected proton was most likely to be found in. In a way, this method amounts to observing the least bound proton of a nucleus under a quantum microscope. By comparing their results with modern shell-model calculations, Olivier and co-workers showed that copper-79 can be best described as a doubly magic nickel-78 nucleus plus a proton added to the next shell above the $Z=28$ gap.

The method used by Welker and colleagues is radically different. Using the most advanced techniques for weighing nuclei, they measured the masses of the chain of copper isotopes from copper-75 to copper-79 at CERN's Isotope Separator On Line Device (ISOLDE) facility. The mass of a nucleus is a direct measure of its ground-state energy, which represents the minimum energy state the nucleus can reach given the underlying interactions between its nucleons. The evolution of the masses along isotopic chains is therefore very sensitive to shell effects and in particular to the occurrence of magic numbers. The authors show results that are compatible with shell-model calculations in which copper-
79 is best described as a single proton on top of a doubly magic nickel-78 core-in agreement with Olivier and colleagues' results.

These two indirect methods provide ample evidence that nickel-78 is indeed doubly magic, but the ultimate proof will come from the direct study of the beast itself. As mentioned, producing nickel-78 in the laboratory is not easy, because most nuclear reactions used to make radioactive nuclei tend to remove neutrons, not add them. Nevertheless, nickel-78 has already been produced, but only in enough quantities to measure its half-life of $122.2 \pm 5.1 \mathrm{~ms}$ [8] - yet another result compatible with its doubly magic nature. More detailed studies of this benchmark nucleus will come with advances in existing and new facilities such as the Facility for Rare Isotope Beams (FRIB) in the US, slated to come online in a few years [9]. One of the first things physicists will try to do using these facilities is to locate nickel-78's first excited state, which is directly related to how magic it really is.

This research is published in Physical Review Letters.

\section{REFERENCES}

[1] M. G. Mayer, "On Closed Shells in Nuclei. II," Phys. Rev. 75, 1969 (1949).

[2] M. G. Mayer, "The Shell Model," Science 145, 999 (1964).

[3] L. Olivier et al., "Persistence of the $Z=28$ shell gap around ${ }^{78} \mathrm{Ni}$ : First spectroscopy of ${ }^{79} \mathrm{Cu}$," Phys. Rev. Lett. 119, 192501 (2017).

[4] A. Welker et al., "Binding Energy of ${ }^{79} \mathrm{Cu}$ : Probing the Structure of the Doubly Magic ${ }^{78} \mathrm{Ni}$ from Only One Proton Away," Phys. Rev. Lett. 119, 192502 (2017).

[5] O. Sorlin and M.-G. Porquet, "Nuclear Magic Numbers: New Features Far From Stability," Prog. Part. Nucl. Phys. 61, 602 (2008).

[6] R. Kanungo et al., "One-Neutron Removal Measurement Reveals ${ }^{24} \mathrm{O}$ as a New Doubly Magic Nucleus," Phys. Rev. Lett. 102, 152501 (2009).

[7] T. Otsuka, R. Fujimoto, Y. Utsuno, B. A. Brown, M. Honma, and T. Mizusaki, "Magic Numbers in Exotic Nuclei and Spin-Isospin Properties of the NN Interaction," Phys. Rev. Lett. 87, 082502 (2001).

[8] Z. Y. Xu et al., " $\beta$-Decay Half-Lives of ${ }^{76,77} \mathrm{Co},{ }^{79,80} \mathrm{Ni}$, and ${ }^{81} \mathrm{Cu}$ : Experimental Indication of a Doubly Magic ${ }^{78} \mathrm{Ni}$," Phys. Rev. Lett. 113, 032505 (2014).

[9] https://frib.msu.edu.

10.1103/Physics.10.121 